\title{
NMDA antagonist MK-801 impairs acquisition but not performance of spatial working and reference memory
}

\author{
MATTHEW L. SHAPIRO and ZOGRAFOS CARAMANOS \\ McGill University, Montreal, Quebec, Canada
}

\begin{abstract}
To assess the effects of NMDA receptor blockade on memory tasks that require the hippocampal system, rats were injected with either MK-801, a noncompetitive NMDA antagonist known to block induction of long-term potentiation in vitro, or normal saline before daily behavioral testing in both working-memory (WM) and reference-memory (RM) procedures on an eight-arm radial maze. Even after 64 days of testing, rats given MK-801 performed poorly on both RM and WM tasks relative to rats given saline. The same dose of MK-801 that impaired memory acquisition affected neither WM nor RM performance in trained rats that had been given saline. In these rats, combined MK-801 and naloxone affected neither WM nor RM, larger doses of MK-801 produced slight ataxia and both WM and RM deficits, but none of the doses produced a selective WM impairment. Interposing a 2-h delay between the first two and the last two correct WM choices produced a small increase in both WM and RM errors, which demonstrates that MK-801 does not selectively impair long-term WM. Finally, rats given MK- 801 chronically and then withdrawn from the drug learned both the WM and the RM tasks, and were subsequently affected only mildly by low doses of MK-801. The results show that functioning NMDA receptors are necessary for learning spatial WM and RM tasks, but not for performing WM or RM tasks after training, and imply that two mechanisms may be required for storing relational (e.g., spatial) memory: an NMDA-dependent mechanism for long-term storage of spatial representations, and a second, nonNMDA mechanism for operating upon those representations.
\end{abstract}

Long-term potentiation (LTP) is a measure of synaptic plasticity that may reflect an important mechanism of information storage in the forebrain (Bliss \& Lynch, 1988; Lynch, 1986; McNaughton \& Morris, 1987). LTP is produced most easily in the hippocampus, a brain structure required for spatial learning and memory (Barnes, 1988). Several classes of experiments have provided converging evidence associating hippocampal LTP and spatial memory in tasks that require the hippocampus: (1) LTP is induced by spatial learning (Green \& Greenough, 1986; Sharp, McNaughton, \& Barnes, 1985); (2) LTP and spatial memory performance both decay more rapidly in aged than in young rats (Barnes \& McNaughton, 1985); and (3) saturating hippocampal LTP prevents spatial learning, which has been interpreted as suggesting that changes in patterns of synaptic strengths in the hippocampus are needed to acquire spatial knowledge (McNaughton, Barnes, Rao, Baldwin, \& Ras-

\footnotetext{
This research was supported by grants to M.L.S. from the Stairs Memorial Fund, McGill University, the Natural Sciences and Engineering Research Council of Canada, and Fonds pour la Formation de Chercheurs et l'Aide à la Recherche, and to Z.C. by the Fonds de la Recherche en Santé du Quebec. The authors thank Michela Gallagher, Norman White, David Olton, Neal Cohen, and Richard Morris for their comments, and Peter Milner, Paul Anderson, and Merck Sharp \& Dohme for their donation of MK-801. Correspondence may be addressed to Matthew L. Shapiro, Department of Psychology, McGill University, 1205 Dr. Penfield Ave., Montreal, Quebec H3A 1B1, Canada.
}

mussen, 1986). These studies are consistent with, but do not prove, the hypothesis that LTP and memory share important common mechanisms.

One such mechanism may be the steps that initiate changes in synaptic efficacy in the hippocampus. LTP induction in hippocampal CA1 and dentate gyrus cells requires the activation of N-methyl-D-aspartate (NMDA) receptors, which are concentrated in $\mathrm{CAl}$ (Collingridge, Kehl, \& McLennan, 1983). However, NMDA receptor activation is not needed for LTP maintenance or expression (Brown, Chapman, Kairiss, \& Keenan, 1988; Muller, Larson, \& Lynch, 1989). The operationally distinct stages of LTP induction, maintenance, and expression appear to be analogous to those traditionally described in memory research-that is, acquisition, storage, and retrievalalthough, clearly, the two differ greatly in level of organization. If induction of LTP in the hippocampus and spatial memory acquisition share common mechanisms, then treatments that block LTP induction should prevent spatial learning. Indeed, intraventricular administration of aminophosphonovaleric acid (AP5), a selective NMDA receptor antagonist, blocks LTP induction and spatial, but not cued, reference-memory (RM) acquisition in the water maze (Morris, Anderson, Lynch, \& Baudry, 1986). Furthermore, like hippocampal lesions, AP5 impairs the acquisition of differential reinforcement of low rates performance (Rawlins, 1985; Tonkiss, Morris, \& Rawlins, 1988) and olfactory discrimination learning (Staubli, 
Thibault, DiLorenzo, \& Lynch, 1989). Together, these studies suggest that LTP induction in the hippocampus and memory acquisition that requires the hippocampus share an NMDA-dependent mechanism.

MK-801 [(+)-10,11-dihydro-5-methyl-5H-dibenzo (a,d) cycloheptene-5,10 imine] is a noncompetitive NMDA antagonist that blocks LTP induction in vitro (Coan, Saywood, \& Collingridge, 1987; Kemp, Priestly, \& Woodruff, 1986; Swartzwelder, Ferrari, Anderson, \& Wilson, 1989) and in vivo (Abraham \& Mason, 1988). Like AP5 and hippocampal lesions, MK-801 impairs place but not cue learning in the water maze, and does not impair spatial memory performance in rats trained before the drug is given (Robinson, Crooks, Shinkman, \& Gallagher, 1989). MK-801 also impairs working-memory (WM) performance in a continuous nonmatching-tosamplc paradigm (Pontecorvo \& Clissold, 1988), which suggests that tasks that require the hippocampus also require the activation of NMDA synapses.

In the present experiments, we further tested the effect of MK-801 on the performance of memory tasks impaired by hippocampal lesions. The working hypothesis was that MK-801 should block the NMDA-dependent induction of synaptic plasticity in the hippocampus and elsewhere, and should thereby impair memory acquisition in tasks requiring the hippocampus. Furthermore, memory performance should not be impaired by MK-801 after learning has occurred, because synaptic plasticity established during learning via NMDA receptors does not require that these receptors function in maintaining or expressing the established plasticity.

The effects of MK-801 were tested in an eight-arm radial maze using two spatial memory tests and two training conditions that are differentially sensitive to hippocampal damage (Olton \& Papas, 1979). In these $4 / 8$ tests, the eight arms of the maze were classified into a baited set and an unbaited set, so that only four of the eight arms ever contained food. To perform the 4/8 task optimally, a rat should enter each baited arm once on each trial and should never enter an unbaited arm. Operationally, performance involves both WM and RM procedures (Olton, Becker, \& Handelmann, 1979). WM procedures require memory for events within a trial (Olton et al., 1979); in the $4 / 8$ task, WM is needed to remember which arms have been entered during a trial. RM procedures require memory for events consistent in all trials; in the 4/8 task, $\mathrm{RM}$ is needed to distinguish the arms in the baited and unbaited sets. Normal rats learn to enter each baited arm once on each trial and avoid entering unbaited arms. Fimbria-fornix lesions impaired WM but not RM performance in rats given extensive preoperative training in a version of the $4 / 8$ task using a 17 -arm maze (Olton \& Papas, 1979). However, fimbria-fornix lesions given before training blocked spatial RM acquisition in operationally similar tasks (Sutherland, Arnold, \& Rodriguez, 1987; cf. Olton, 1983; for a review, see Barnes, 1988).

These two spatial memory tasks (WM and RM) and two testing conditions (acquisition and retention) provide strong tests of the hypothesis that NMDA blockade impairs learning but not performance in tasks that require the hippocampus. If NMDA receptor function is necessary for learning but not for performance, then MK-801 should (1) impair both spatial WM and RM acquisition in naive animals; (2) not impair spatial RM performance in trained animals, because synaptic plasticity should be required only for acquiring but not for expressing stored information; and (3) impair spatial WM performance in trained animals, because the information needed for WM performance must be acquired during each trial, if this type of memory acquisition uses the same mechanism as that used by RM.

\section{GENERAL METHOD}

\section{Subjects}

Sixteen female Sprague-Dawley rats, weighing 230-270 $\mathrm{g}$ at the start of testing, were housed individually on a 12:12 h light:dark cycle with water always available. Each rat was pseudorandomly assigned to either an MK group (and given MK-801 during acquisition) or a SAL group (and given saline during acquisition) (see Experiment 1, below). One of the SAL rats stopped entering arms during testing and was removed from the experiment.

\section{Apparatus}

An eight-arm radial maze was used for testing spatial memory. An octagonal central platform $40 \mathrm{~cm}$ across was elevated $60 \mathrm{~cm}$ above the floor. One maze arm, $55 \mathrm{~cm}$ long and $9 \mathrm{~cm}$ wide, with edges $2 \mathrm{~cm}$ high, extended from each of the eight sides of the central platform. The central platform and maze arms were made of wood and painted gray. Guillotine doors made of clear Plexiglas controlled access to each arm. Food wells were drilled $0.5 \mathrm{~cm}$ deep, $2 \mathrm{~cm}$ from the end of each arm. The maze room contained windows, posters, tables, a radio, and other extramaze stimuli. An opaque cloth curtain, $2 \mathrm{~m}$ high, was used to reduce extramaze stimuli during probe tests described below.

\section{Behavioral Testing}

Shaping. The rats were food-deprived to $85 \%$ of their ad-lib body weights before shaping, and were thereafter given enough rat chow (Purina) daily to maintain normal growth. Each rat was gentled by handling for $15 \mathrm{~min}$ daily for several days. The rats were then shaped to enter the arms of the maze. On Day 1 of shaping, Froot Loops (Kellogs; Rexdale, Ontario) were scattered on the maze, and the rats were placed, 4 at a time, on the maze for $15 \mathrm{~min}$. On 6 subsequent days, 1 Froot Loop was placed in the food well at the end of each arm, and each rat was put on the maze alone for $10 \mathrm{~min}$ or until all of the Froot Loops were eaten. To habituate the rats to daily injections, each rat was injected i.p. with $0.2 \mathrm{ml}$ of normal saline $30 \mathrm{~min}$ prior to being put in the maze.

The 4/8 task. Before each trial, one Froot Loop was placed in the food cup of each of the four baited arms. Each rat in the MK group was assigned a unique set of baited and unbaited arms, which was consistent throughout the experiment. Each rat in the SAL group was tested with the same pattern as 1 of the MK rats. For each trial, WM errors were counted when a rat entered an arm of the baited set more than once, and RM errors were counted when a rat entered an arm of the unbaited set (adapted from Olton \& Papas, 1979).

At the start of each trial, a rat was put onto the central platform with the guillotine doors closed. The eight doors were then raised. A choice was noted when the rat's hindquarters passed the door to an arm, and all doors except the one to the entered arm were then lowered. When the rat returned to the central platform, the open door was closed. After a 5-10-sec delay, all of the doors were 
opened to allow another arm entry. This procedure was repeated until all four of the baited arms had been chosen or $10 \mathrm{~min}$ had elapsed. The maze was sponged clean daily after all rats were tested.

\section{Experimental Design}

Experiments were designed to test specific hpotheses relating NMDA receptor function to memory performance. Four experiments were executed. In Experiment 1, the effects of MK-801 on WM and RM acquisition in the 4/8 task was tested by comparing the initial acquisition performance of rats given either MK-801 or saline 30 min before each trial. Long-term behavioral impairments produced by chronic MK-801 administration were tested by extensive training and MK-801 withdrawal. The rats were tested with MK-801 for 64 trials (the prolonged MK testing period) and were then given saline for an additional 40 trials (the withdrawal phase). In Experiment 2, spatial probes assessed the stimuli used by SAL rats to discriminate between baited and unbaited arms. In Experiment 3 , the rats that had received saline during training were given MK-801, and retention and drug probes assessed the effects of MK-801 on previously learned spatial memory performance. Probes further investigated the effects of different doses of MK-801 and the opiate antagonist naloxone. In Experiment 4, a 2-h delay between the first two correct and the last two correct WM choices was imposed to assess whether MK-801 impaired long-term WM.

\section{EXPERIMENT 1 INITIAL ACQUISITION}

Spatial WM and RM acquisition are impaired by hippocampal lesions (Barnes, 1988). If NMDA receptor function is necessary for acquiring spatial knowledge, then MK-801 should impair the acquisition of both WM and RM tasks.

\section{Method}

The 16 rats were pseudorandomly assigned to either an MK group or a SAL group. Thirty minutes before testing, the rats were injected i.p. with either MK-801 (the MK group, $n=8$; $0.0625 \mathrm{mg} / \mathrm{kg}$ ) or an equivalent volume of normal saline (the SAL group, $n=8$ ). The dose of MK-801 was chosen after informal observations of various doses of the drug. The present dose produced no observable behavioral effects other than mild hyperactivity, which appeared to be similar to that produced by fornix lesions. Acquisition performance for each rat was tested once daily for 34 days until the SAL rats had reached criterion performance (the initial acquisition period). The rats in the MK group were subsequently given MK-801 for an additional 30 daily trials to assess the longterm effects of the drug (the prolonged MK testing period; Figure 1). Any long-term effects of MK-801 could be produced by continuing drug effectiveness, by damage produced by long-term MK-801 administration (Olney, Labruyere, \& Price, 1989), or both. To distinguish among these possibilities, the MK rats were then given saline for $\mathbf{4 0}$ trials (the withdrawal phase). The SAL rats were tested in the probe tests described below during this postacquisition period.

\section{Results}

MK-801 impaired the acquisition of both WM and RM in the 4/8 task (Figures $1 A$ and 1B). After 15 trials, the SAL rats made a mean of less than $1 \mathrm{WM}$ error, whereas the MK rats made a mean of 3 or more WM errors per trial. Even after 34 days (seven blocks) of training, the MK rats were severely impaired relative to the SAL rats [MANOVA, interaction of groups $\times$ the first seven blocks of repeated trials, $F(6,8)=10.55, p<.003$; ANOVA, main effect of groups, MK vs. SAL, $F(1,13)=32.99$, $p<.001$; Block $7, F(1,13)=9.75, p<.01]$. The SAL rats gradually acquired the RM task, so that after 30 trials they made about 1 RM error per trial. However, the MK rats showed no evidence of learning the $R M$ discrimination and made almost 3 RM errors per trial at this time [MANOVA, interaction of groups $\times$ the first seven blocks of trials, $F(6,8)=3.92, p<.05$; ANOVA, main effect of groups, MK vs. SAL, $F(1,13)=82.8, p<.001$; Block 7, $F(1,13)=26.12, p<.001]$. In addition to impairing memory, MK-801 produced general changes in behavior that resembled the effects of hippocampal-system lesions. The MK rats entered the arms of the maze more quickly than did the SAL rats, which showed some disinhibition. The MK rats also tended to re-enter the maze arms they had just exited, a perseverative behavior never observed in the SAL rats.

WM performance improved gradually in the MK rats given extensive training, and improved during the first block of saline trials after MK-801 withdrawal, but did not improve for the remaining eight blocks of saline trials [Figure 1A; WM acquisition by MK rats only during Blocks 1-13 of testing, repeated measures ANOVA by blocks, $F(12,84)=6.4, p<.001$; linear trend, $F(1,7)=$ $57.7, p<.001$; withdrawal of MK-801, Block 13 vs. Withdrawal Block $1, F(1,7)=4.66, p=.07]$. After 64 drug trials and 30 saline trials, the MK rats made, on average, less than $0.5 \mathrm{WM}$ errors per trial, but they did not perform the WM task as well as the SAL rats had after only 30 trials [Figure 1A, SAL Blocks 6 and 7 vs. MK Withdrawal Blocks 7 and $8, F(1,13)=19.26, p<.002$ ]

The WM performance of the MK group was highly variable (Figure 1A). To investigate the source of this variability more closely, the MK group was divided into two subgroups composed of the 4 best ("tolerant") and 4 worst ("nontolerant") WM performers based on the eighth block of trials (Figure 1C). ${ }^{1}$ The WM performance of the two subgroups differed over the entire 13-block acquisition period, with no difference in acquisition curves [ANOVA comparing MK subgroups, $F(1,6)=10.73$, $p<.02$; interaction of subgroups $\times$ days, $F(12,72)=$ $0.53, p<.89$ ]. MK-801 impaired WM acquisition in both subgroups, but the tolerant rats in the MK group performed the WM task with a mean of only 1 error after 30 trials (Block 6), whereas the nontolerant rats committed a mean of 3.6 errors at that time in training. However, the tolerant rats were impaired relative to the saline group [ANOVA, MK tolerant rats vs. SAL group, $F(1,9)=$ $39.1, p<.001$; MANOVA of groups $\times$ blocks, $F(6,4)=16.5, p<.01]$. The performance of both subgroups stopped improving by Block 10 of training. After withdrawal, the tolerant rats' performance did not change, whereas the nontolerant rats' performance improved dramatically, but not significantly, from 3.06 to $0.7 \mathrm{WM}$ errors [MANOVA by trials, Block 13 vs. Withdrawal Block $1, F(1,3)=6.62, p<.082]$.

RM performance by the MK rats did not improve even during extensive training [repeated measures ANOVA by blocks, $F(12,84)=1.41, p=.18$; Figure 1B]. Note that 

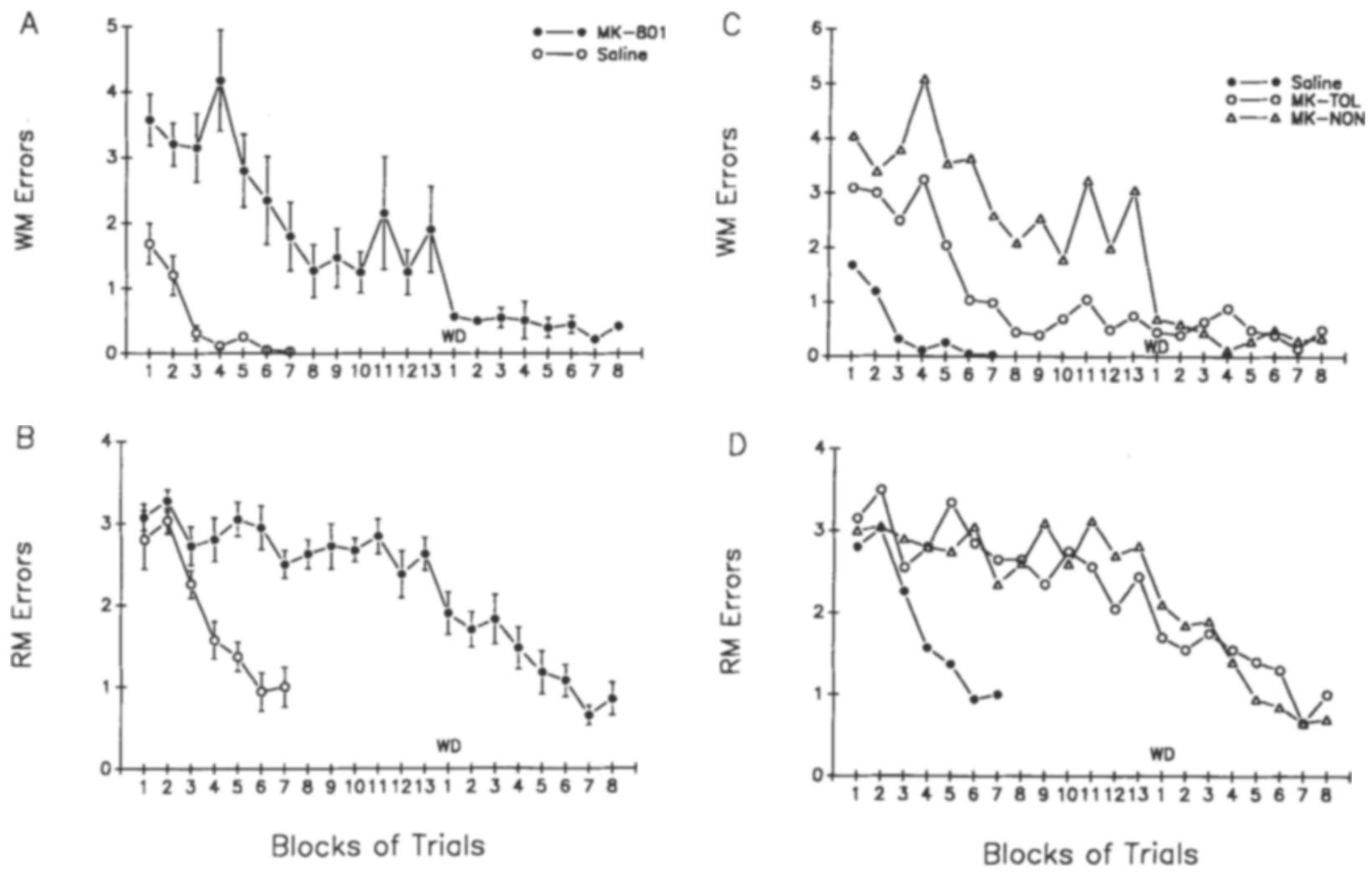

Figure 1. MK-801 impairment of spatial learning. Each circle represents the mean errors $( \pm 1 S E M)$ in a block of 5 days of testing, except for Blocks 7 and 13, which represent 4 days. Rats in both groups were tested for the first seven blocks. Testing with MK-801 continued for the MK group for five more blocks (blocks 8-13). The withdrawal (WD) of MK-801 is indicated after Block 13, and drugfree testing continued for eight blocks. (A) Working-memory (WM) performance was impaired throughout the acquisition period by MK-801, and prolonged exposure to MK-801 produced a small but enduring long-term behavioral deficit in WM after withdrawal. (B) Referencememory (RM) acquisition was blocked completely by MK-801 throughout drug testing. Withdrawal of MK-801 produced a gradual improvement in RM performance to normal levels. (C) One subgroup of rats (MK-TOL) appeared to tolerate the effects of MK-801 on WM better than the other subset (MK-NON). (D) The two subsets of MK rats that were differentially impaired in WM acquisition performed similarly in RM acquisition.

the variance in the RM performance of the MK group is quite small. Indeed, the subgroups that appeared to be tolerant or intolerant to MK-801 in WM performed identically in RM (Figures 1C and 1D). RM improved gradually after MK-801 withdrawal, so that the MK rats performed within the normal range after about 30 drug-free trials (Figure 1B) [repeated measures ANOVA, Withdrawal Blocks $1-8, F(7,49)=6.54, p<.001]$. The rate of acquisition after withdrawal did not differ significantly from that in the SAL group during the original acquisition period [MK Withdrawal Blocks 1-7 vs. SAL Acquisition Blocks 1-7, repeated measures MANOVA, groups $\times$ blocks, $F(6,8)=2.6, p=.1]$.

\section{Discussion}

MK-801 administered 30 min before testing impaired the acquisition of spatial WM and RM performance in the radial maze. These results are consistent with others showing that AP5 and MK-801 impair spatial memory acquisition (Morris et al., 1986; Robinson et al., 1989). The individual differences in WM performance shown by the MK rats is similar to a variable duration of efficacy in clinical trials, in which individual differences in responses to the antiseizure effects of MK-801 have also been reported (Troupin, Mendius, Cheng, \& Risinger, 1986; also see General Discussion, below). These results, together with the effects of LTP saturation in the hippocampus and lesions of the hippocampal system, corroborate the hypothesis that NMDA receptor activation is a critical part of a mechanism of memory acquisition associated with the hippocampus, presumably because of the role of NMDA receptors in the induction of synaptic plasticity (McNaughton \& Morris, 1987; Robinson et al., 1989). However, because of the association of WM and RM errors, the current results are also consistent with many other possible effects of MK-801, such as impairments in perception, motivation, attention, arousal, motor performance, and many other nonspecific, nonmnemonic brain processes (but see Experiment 3 below).

\section{EXPERIMENT 2 SPATIAL PROBE TESTS}

NMDA antagonists impair the acquisition of RM tasks that are dependent upon extramaze, but not intramaze, cues (Morris et al., 1986; Robinson et al., 1989). The 
RM learning impairment produced by MK-801 shown in Experiment 1 is consistent with these findings. In Experiment 2, we used spatial probe tests to examine whether the present testing environment encouraged the use of extramaze cues to discriminate baited from unbaited arms. After acquisition testing, four probe tests assessed the stimuli used by the SAL rats to discriminate between the baited and unbaited sets (the MK rats were given extended acquisition testing at this time, so the probe tests assessed performance of the SAL rats only). Because previous results have suggested that normal rats use extramaze stimuli to solve this discrimination, hypotheses were tested using directional statistics (Olton \& Samuelson, 1976).

\section{Method}

The food on demand (FOD) probe tested whether the rats could detect the presence of a food pellet at the end of a baited arm. Food was not placed in the food cups until the rat entered an arm of the baited set. If a rat selected arms by detecting the presence of the food pellet, the FOD test should increase errors.

The maze rotation (ROT) probe tested whether the rats used other intramaze cues. The maze was baited and then rotated $135^{\circ}$ clockwise at the beginning of testing. Thus, food pellets were now in locations rotated relative to the room, but in locations consistent with intramaze cues. If a rat selected baited arms on the basis of intramaze cues, then it should enter the arms containing food (which could now be an RM error). However, if a rat selected arms on the basis of their room location, then it should enter the appropriate, though now unbaited, arms in the ROT test.

The curtain (CURT) test assessed the effects of extramaze cues. An opaque curtain encircled the maze and a radio usually playing in the background was silenced. If a rat selected baited arms on the basis of visible extramaze cues or the sound of the radio, then the CURT test should impair performance.

The rotation and curtains ( $R \& C$ ) probe tested whether static background cues (those unaffected by maze rotation, curtains, etc.; O'Keefe \& Speakman, 1987) could be used by the rats to select baited arms. The maze was rotated and the curtain was raised during the same trial. If a rat selected arms by using intramaze cues or extramaze cues that were altered by the curtain, then the $\mathrm{R} \& \mathrm{C}$ test should impair performance. However, if a rat used static background cues, then the R \& $\mathrm{C}$ test should not impair RM performance.

\section{Results and Discussion}

The SAL rats tended to use extramaze cues to discriminate between baited and unbaited arms (Figure 2). The CURT probes produced significantly more errors than occurred in normal trials, whereas neither the FOD nor the ROT probes did so [repeated measures MANOVA, Block 7 vs. CURT trials, $F(1,6)=4.44, p<.05$, onetailed]. Three of the 8 rats were unimpaired during the $R$ \& $C$ probes, which suggests that these rats used static background cues to discriminate the arms (O'Keefe \& Speakman, 1987). WM was not impaired by any of the probe tests, which suggests that the rats used any available cues to remember which arms had been visited on a trial (Figure 2). Thus, whereas the RM discrimination depended upon extramaze stimuli, the WM discrimination did not depend completely upon any definable set of stimuli. Because the spatial probes were designed to determine how the rats discriminated baited from unbaited

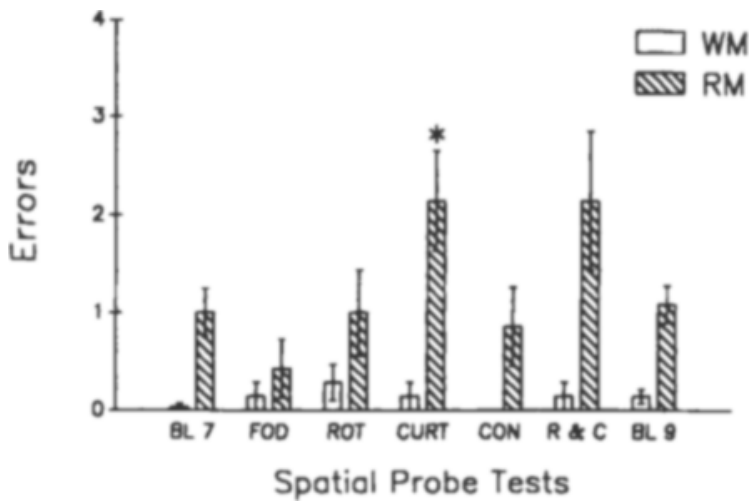

Figure 2. Extramaze stimuli tended to allow discrimination between baited and unbaited arms by saline-treated (SAL) rats. Bars represent mean errors $\pm 1 S E M,{ }^{*}$ indicates a statistically significant difference between a normal block of trials and a probe trial $(p<.05)$. In this figure only, the * indicates a significant directional (one-tailed) test. BL $7=$ the seventh block of acquisition testing, FOD $=$ food on demand, ROT $=$ maze rotation of $135^{\circ}$ clockwise, CURT = the curtains probe, $C O N=$ a control trial, $\mathrm{R} \& \mathrm{C}=$ the maze rotation and curtains probe, and BL $9=$ the ninth block of testing.

arms (i.e., RM discrimination), all probe tests involved altering the maze before the start of a trial. Since none of the probes involved altering the relationships between intra- and extramaze cues during a trial, the probe tests did not distinguish WM choices based on intramaze cues and static background cues. These results show that the SAL rats tended to use extramaze stimuli to discriminate the baited and unbaited arms, and if the same strategies were used by the MK rats, the present results imply that the impairment in RM produced by MK-801 is similar to that found in related studies (Morris et al., 1986; Robinson et al., 1989). ${ }^{2}$

\section{EXPERIMENT 3 \\ RETENTION AND DRUG PROBE TESTS}

\section{Experiment 3A Retention}

If MK-801 selectively impairs memory acquisition by blocking the induction, but not the maintenance or the expression, of NMDA-dependent synaptic plasticity, then the spatial RM performance of well-trained animals should be unimpaired by MK-801 at doses that impair learning (Robinson et al., 1989). In contrast, spatial WM is impaired by hippocampal lesions, and requires daily reacquisition of spatial knowledge (i.e., memory for the specific arms entered at a given point in a trial; Olton, 1983). If MK-801 produces the same deficits in spatial memory as do hippocampal lesions, then MK-801 should impair WM even in well-trained animals. Finally, if MK-801 impairs performance through nonspecific mechanisms (e.g., perception, arousal, motor performance, etc.), then the drug should impair both RM and WM during retention tests. 


\section{Method}

To test the effects of MK-801 on memory retention, the SAL rats from Experiment 1 were given the same treatment that the MK group had been given during acquisition. Because $0.0625 \mathrm{mg} / \mathrm{kg}$ MK-801 seemed to block learning completely in the MK group, we suspected that this dose produced nonspecific performance impairments. To test this possibility, 4 of the 8 rats were given a lower dose $(0.05 \mathrm{mg} / \mathrm{kg})$, and the remaining 4 received the standard dose $(0.0625 \mathrm{mg} / \mathrm{kg})$ of MK-801, injected i.p. $30 \mathrm{~min}$ prior to testing for 10 days. All other procedures were identical to those used during acquisition testing. During the drug probe tests, only the SAL rats were used, and the tests were given during the same period that the MK rats were given extensive acquisition training. The last block of saline trials (SAL Block 9) was given after the spatial probe tests and was used to assess drug effects.

\section{Results and Discussion}

The same dose of MK-801 that had impaired both WM and RM acquisition in the MK rats in Experiment 1 had no measurable effect on either WM or RM retention performance in the SAL group (Figure 3, compare bars marked SAL BL 9 and MK RET). The 0.05- and $0.0625-\mathrm{mg} / \mathrm{kg}$ doses had equivalent effects, and the data were therefore combined for statistical tests [MANOVA, SAL Block 9 vs. MK retention test, WM, $F(1,6)=1.41$, $p=.28 ; \mathrm{RM}, F(1,6)=2.64, p=.15]$.

The lack of impairment in RM retention is consistent with the effects of hippocampal lesions in similar tests (Ol-

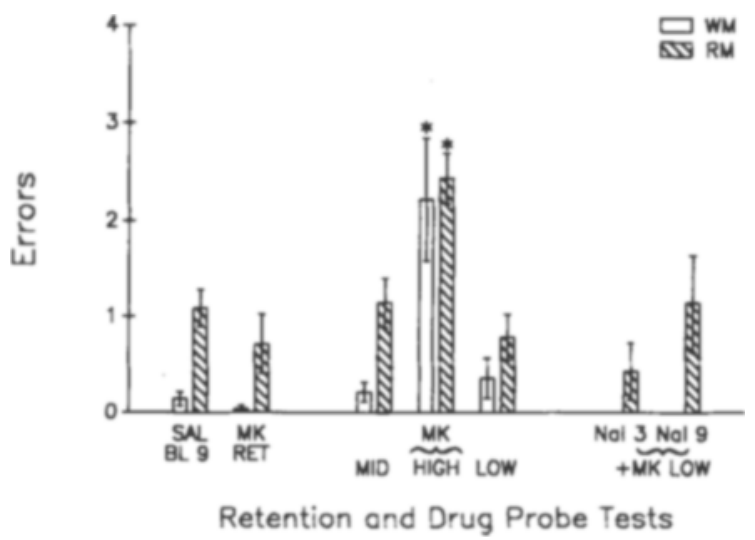

Figure 3. MK-801 did not impair spatial memory performance in trained rats. MK-801 was given to saline-treated (SAL) rats only. Doses are in $\mathrm{mg} / \mathrm{kg}$, injected i.p. Bars represent mean errors \pm 1 $S E M$, * indicates a statistically significant difference between a normal block of trials and a probe trial $(p<.05)$. From left to right, SAL BL 9 = ninth block of acquisition testing, MK RET = the mean of a 10-trial retention test with either a $0.05-$ or a $0.0625-\mathrm{mg} / \mathrm{kg}$ dose of MK-801. MID, HIGH, and LOW are defined in the text. Nal 3 and Nal 9 are each the mean of two probe trials in which MK-801 and naloxone were both given to the rats. On the retention tests (SAL BL 9 vs. MK RET), the same dose of MK-801 that had impaired learning in the MK group during acquisition had no effect when given to SAL rats. On MK-801 probe tests (SAL BL 9 vs. MID, HIGH, and LOW), only the high dose of MK-801 produced significant performance deficits, and this dose increased both workingmemory (WM) and reference-memory (RM) deficits relative to saline, LOW, or MID tests. On naloxone probe tests (SAL BL 9 vs. Nal 3 and Nal 9), naloxone in combination with MK-801 did not significantly affect performance. ton \& Papas, 1979) and of the NMDA antagonists AP5 (Morris et al., 1986) and MK-801 (Robinson et al., 1989) in the Morris water maze. However, because hippocampal lesions impair spatial WM retention performance in the 4/8 task (Olton \& Papas, 1979), the lack of an effect of MK-801 on WM was not predicted (Figure 3, nonhatched bars). Somewhat analogous results were obtained when LTP was saturated in the dentate gyrus by prolonged and repeated tetani delivered to the perforant path: rats were impaired in acquiring spatial RM but were unimpaired in the standard eight-arm radial maze (McNaughton et al., 1986). WM requires information storage for the animal to remember which arms have been visited during each daily trial. Therefore, the normal WM performance in well-trained rats given MK-801 suggests that WM information storage is different in some way than that required for RM acquisition.

\section{Experiment 3B \\ MK-801 Dose-Response Tests}

If WM in trained rats depends on NMDA receptor function, but to a lesser extent than that required for RM acquisition, then higher doses of MK-801 should produce selective WM impairments.

\section{Method}

The rats in the SAL group that were tested in the retention experiments were given higher doses of $\mathrm{MK}-801$, in $0.025-\mathrm{mg} / \mathrm{kg}$ increments. The doses spanned a range to test whether any concentration could be found to selectively block WM. Four of the $8 \mathrm{SAL}$ rats were given $0.075 \mathrm{mg} / \mathrm{kg}$, the remaining 4 were given $0.10 \mathrm{mg} / \mathrm{kg}$ MK-801, for two daily trials. The same rats were then given either 0.125 or $0.15 \mathrm{mg} / \mathrm{kg} \mathrm{MK-801}$, and 0.05 and $0.0625 \mathrm{mg} / \mathrm{kg}$ MK-801 during each of two additional probe trials. The $0.075-$ and $0.1-\mathrm{mg} / \mathrm{kg}$, the $0.125-$ and $0.15-\mathrm{mg} / \mathrm{kg}$, and the 0.05 - and $0.0625-\mathrm{mg} / \mathrm{kg}$ dosage pairs did not produce different effects, and thus were combined into mid, high, and low dose ranges for statistical purposes. The low doses were given to determine if the high doses of MK-801 produced an impairment that endured beyond the 2 days of actual administration. Each dose was given twice in separate daily trials to each animal, with a saline trial between each MK-801 trial. All other aspects of the procedure were the same as those during the acquisition and retention tests.

\section{Results and Discussion}

No dose of MK-801 selectively impaired WM (Figure 3, MK MID, HIGH, LOW). Only the high doses of MK-801 impaired WM performance in the well-trained SAL rats. However, these high doses of MK-801 impaired RM performance similarly (Figure 3) [SAL Block 9 vs. high, WM, $F(1,6)=13.37, p<.012 ; \mathrm{RM}, F(1,6)=$ 56.64, $p<.001$; mid vs. high, RM, $F(1,6)=74.8$, $p<.001 ; \mathrm{WM}, F(1,6)=11.2, p<.02]$. Neither the mid nor the low doses impaired either WM or RM.

The lack of dissociation of WM and RM suggests that the impairment produced by high doses of MK-801 were not specific to memory. Indeed, the high doses produced mild ataxia, head weaving, and transient aphagia in addition to the memory deficits. Similar nonspecific effects of high doses of MK-801 have been reported elsewhere 
(e.g., Pontecorvo \& Clissold, 1988; Robinson et al., 1989). These results, together with the findings from the acquisition and retention experiments, further suggest that short-term spatial WM performance in trained rats does not require NMDA-dependent synaptic plasticity.

Because hippocampal lesions impair spatial WM in well-trained rats, but NMDA blockade does not, a critical question remains about the mechanisms of WM. Perhaps two pharmacologically distinct mechanisms for information storage involve the hippocampus: one mechanism involving NMDA receptors and enabling a form of synaptic plasticity required for acquiring spatial knowledge, and a second, NMDA-independent mechanism, which may be required for storing WM information. This WM storage could involve an NMDA-independent form of synaptic plasticity.

\section{Experiment 3C Naloxone Tests}

One form of NMDA-independent plasticity in the hippocampus has been reported in the mossy fiber-CA3 synapse (Harris \& Cotman, 1986), which is reportedly blocked with the opiate antagonist naloxone (Derrick \& Martinez, 1988). Although naloxone alone does not impair spatial WM, and indeed may improve radial maze performance (Gallagher, 1985), opiate- and NMDAdependent synaptic plasticity may work in parallel to subserve WM. If WM information is stored with either NMDA- or opiate-dependent plasticity, then functionally blocking both NMDA and opiate synapses should impair WM performance. However, for reasons cited above, RM performance should not require synaptic plasticity.

\section{Method}

Each SAL rat was given two trials that combined naloxone (either 3 or $9 \mathrm{mg} / \mathrm{kg}$ ), administered $15 \mathrm{~min}$ before testing, with MK-801 $(0.0625 \mathrm{mg} / \mathrm{kg})$, administered $30 \mathrm{~min}$ before testing. Injecting $3 \mathrm{mg} / \mathrm{kg}$ naloxone i.p. produces a complete but relatively selective opiate receptor blockade, whereas $9 \mathrm{mg} / \mathrm{kg}$ produces considerable nonspecific effects (Sawynok, Pinsky, \& La Bella, 1979). The rats in the SAL group were tested with naloxone and MK-801 for two trials.

\section{Results and Discussion}

Neither dose of naloxone in combination with MK-801 impaired either WM or RM (Figure 3, Nal 3 and Nal 9). Rather, as reported elsewhere, naloxone appeared to improve WM performance (Gallagher, 1985), although this was not a significant effect here [SAL Block 9 vs. Nal 3, WM, Wilcoxon $z=-1.63, p=.102$; RM, $F(1,6)=$ $2.614, p=.16$; SAL Block 9 vs. Nal 9, WM, Wilcoxon $z=-1.63, p=.102 ; \mathrm{RM}, F(1,6)=0.014, p=.91] .^{3}$ Thus, combined blockade of opiate and NMDA receptors did not impair WM performance. These results show that if LTP in CA3 is opiate-dependent, it is not a likely candidate to store WM information in trained rats given MK-801. Thus, neither of two reported forms of LTP in the hippocampus appears to be responsible for storing WM information for short periods. ${ }^{4}$

\section{EXPERIMENT 4 LONG-TERM WORKING MEMORY}

The amnestic effects of hippocampal lesions are often revealed when a delay is interposed between the initial exposure to information and the test requiring that knowledge. This effect is shown in delayed matching-to-sample or delayed nonmatching-to-sample tests, in which memory impairment is clearly related to the amount of time an animal must remember information (Dunnett, 1985; Mishkin, 1982). LTP is often defined as potentiation that persists for several hours, to distinguish it from other forms of potentiation (Baudry, Larson, \& Lynch, 1988). Perhaps WM performance requires LTP mechanisms when WM information is to be stored for prolonged periods. If LTP induction is required for acquiring long-lasting $W M$, then forcing rats to remember WM choices over a delay period should increase errors in animals given treatments that block LTP induction.

To evaluate the effects of MK-801 on WM more fully, long-term WM was tested. In this test, each rat was placed on the maze and allowed to enter two of the baited arms (the sample phase). The rat was then placed in a holding cage for $2 \mathrm{~h}$ (the delay phase) and was then put back in the maze and allowed to enter arms until the remaining baited arms were entered or $10 \mathrm{~min}$ had elapsed (the choice phase). If storing long-term WM in the radial maze task requires NMDA-dependent synaptic plasticity, then administering MK- 801 before the sample phase should produce time-dependent WM errors. Specifically, MK-801 should increase retrograde WM errors: during the choice phase, the rats should re-enter the arms they had chosen during the sample phase. However, MK-801 should not produce anterograde WM errors: during the choice phase, the rats should not re-enter arms chosen during that phase, because little time would have elapsed between such choices.

\section{Method}

This test was performed after the withdrawal phase of testing of the MK rats. The rats from both the SAL and the MK groups were injected with saline $30 \mathrm{~min}$ before being placed in the maze, and were given one trial daily in the long-term WM task until each rat made no more than 1 error (WM and RM combined) for two consecutive trials. On test trials, each rat was injected with either MK-801 $(0.0625 \mathrm{mg} / \mathrm{kg})$ or an equal volume of saline $30 \mathrm{~min}$ before it was placed in the maze for the first two choices. Each of three MK-801 trials was separated by at least one saline trial to ensure stable performance in this within-subjects test.

\section{Results and Discussion}

The rats learned to perform the long-term WM task nearly perfectly after a mean of 10 trials (Figure 4A). MK-801 produced a small but statistically significant impairment in both WM and RM in the 2-h tests [WM, $F(1,14)=5.32, p<.05 ; \mathrm{RM}, F(1,14)=24.01$, $p<.001]$. WM errors increased from a mean of 0.12 during saline trials to 0.53 during MK-801 trials. Virtually all WM errors occurred after the delay in both the saline and the drug trials. However, neither retrograde 

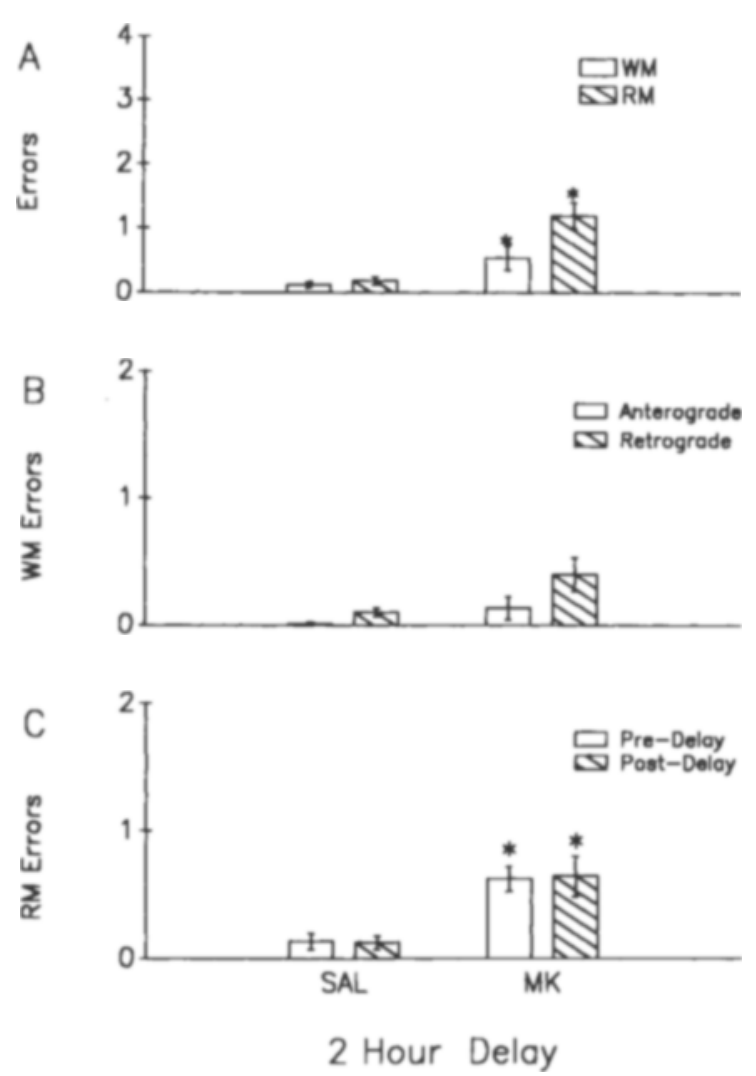

Figure 4. Delay probe test. (A) MK-801 produced an increase in both working-memory (WM) and reference-memory (RM) errors when a 2-h delay was interposed between the first two correct and the last two correct WM choices. (B) Neither anterograde nor retrograde WM errors increased statistically, although the increase in retrograde errors approached significance. (C) MK-801 produced the same degree of RM impairment before and after the 2-h delay.

nor anterograde errors increased significantly during MK-801 trials relative to saline trials when considered separately, although retrograde errors approached significance [Figure 4B; saline vs. MK-801, anterograde WM errors, $F(1,14)=2.30, p=.15$; retrograde WM errors, $F(1,14)=4.55, p=.051$; interaction of drug and error type, $F(1,14)=1.77, p=.21]$.

RM errors increased from a mean of 0.19 during saline trials to 1.19 during MK-801 trials. RM errors occurred with equal frequency during the sample and test phases, which indicates that the drug produced a nonspecific impairment in both phases of the delay task [Figure 4C; saline vs. MK-801 treatment, predelay, $F(1,14)=16.9, p<.002$; postdelay, $F(1,14)=10.18$, $p<.008]$. The increase in both WM and RM errors on the delay test is curious in light of the absence of deficits produced by MK-801 during the retention tests. If MK-801 blocked only the induction of synaptic plasticity, then the drug should not have affected RM performance. Several factors may be relevant here. The actual number of RM errors produced by MK-801 in the present test was within the range of normal performance during acquisition and retention testing (compare Figures $4 \mathrm{~A}$ and $1 \mathrm{~B}$,
Blocks 7 and 9). Thus, the rats performed worse during the drug probe tests than they did during the present test, and the improved baseline performance here may have revealed a deficit produced by the drug. Finally, both MK and SAL rats were given this test, and chronic drug treatment may have increased the efficacy of the drug. Although this trend appeared when the errors of the MK and SAL rats were compared, it was not significant (Table 1). Thus, MK rats, which received MK-801 chronically, were not more susceptible to the drug during probe tests than were the SAL rats.

Together, the effects of MK-801 on delay tests do not provide strong evidence that NMDA receptor activation is required for storing long-term WM. Thus, information about entered arms was maintained for $2 \mathrm{~h}$ despite the ostensible blockade of NMDA function. These results suggest that WM may be independent of NMDA receptor function even after a 2 -h delay in extensively trained rats. Nonetheless, the increase in retrograde errors produced by MK-801 approached significance, and may prove to be reliable with repeated tests. Of course, more difficult WM tasks, or delays longer than $2 \mathrm{~h}$, may reveal an amnestic effect of MK-801 on WM.

\section{GENERAL DISCUSSION}

Low doses of MK-801 administered at the time of testing impaired learning but spared retention performance of spatial RM and WM in the $4 / 8$ radial maze task. These experiments confirm and extend previous findings showing that NMDA receptor blockade impairs spatial memory acquisition but not performance (Morris et al., 1986; Robinson et al., 1989). As might be predicted by a hypothesis linking synaptic plasticity induction to memory acquisition, MK-801 produced a learning deficit even with more than 2 months of chronic administration and testing, but the amnestic effects were greatly attenuated af-

Table 1

Mean $(₫ S E M)$ Baseline Errors as a Function of Chronic MK-801 Administration

\begin{tabular}{lllll}
\hline & \multicolumn{5}{c}{ Injection } \\
\cline { 2 - 5 } Group & \multicolumn{2}{c}{ Saline } & \multicolumn{2}{c}{ MK-801 } \\
\cline { 2 - 5 } \cline { 3 - 4 } & $M$ & $S E M$ & $M$ & $S E M$ \\
SAL & 0.09 & 0.04 & 0.24 & 0.11 \\
MK & 0.15 & 0.08 & 0.79 & 0.29 \\
\multicolumn{5}{c}{ Working-Memory Errors } \\
SAL & \multicolumn{7}{c}{ Reference-Memory Errors } \\
MK & 0.17 & 0.07 & 1.00 & 0.38 \\
\hline
\end{tabular}

Note-SAL $=$ rats treated with saline prior to training on the workingmemory (WM) and reference-memory (RM) tasks, MK = rats treated with MK-801 prior to training on the WM and RM tasks. Performance of MK and SAL rats on the long-term WM task are analyzed separately. Although WM errors appear to increase more in rats exposed to chronic MK-801, the interaction between groups and drug treatment was not significant [MANOVA, $F(1,13)=1.98, p=.183$ ]. A similar trend of even smaller magnitude appears in RM errors. 
ter a brief period of drug-free training. Thus, MK- 801 did not damage memory systems, but prevented memory acquisition. MK-801 did not produce a significant retrograde WM impairment when a 2-h delay was imposed between the sample and choice phases, which suggests that spatial WM storage in trained rats may require a different mechanism than does NMDA-dependent synaptic plasticity. Furthermore, the combination of MK-801 and naloxone, which should have blocked the two putative mechanisms of long-term synaptic plasticity induction in the hippocampus, did not impair WM. Each of these results is discussed below, within a framework that assumes that MK-801 impairs memory by blocking synaptic plasticity in the hippocampus.

\section{Acquisition versus Retention}

The dissociation between acquisition and retention of spatial memory performance demonstrates that the acquisition deficit was not due to nonspecific effects of MK-801, but rather to a selective effect of the drug on learning. This dissociation corroborates previous findings that MK-801 and AP5 impair memory acquisition but not retention performance (Robinson et al., 1989; Staubli et al., 1989). Together with LTP studies, the present results provide additional evidence that NMDA receptor activation is a critical step both for inducing LTP and for acquiring spatial memory (Morris et al., 1986). Although other studies have suggested that the critical synapses for spatial memory storage exist in the hippocampus, the present study involved systemic drug administration and did not test hippocampal LTP, and thus did not address this issue.

\section{Long-Term Effects of MK-801}

Chronic MK-801 treatment produced an enduring RM impairment that lasted until drug withdrawal. In contrast, WM improved gradually during MK-801 treatment, and improved more quickly upon drug withdrawal. This finding further supports the notion that two mechanisms of memory storage exist, one required for RM acquisition and another for WM acquisition. A small impairment in WM outlasted the acute effects of drug administration (Figure 1A). These behavioral changes may reflect the neurotoxic effect of MK-801 reported elsewhere (Olney et al., 1989). However, no significant increase in sensitivity to the effects of MK-801 occurred during the longterm WM test (Table 1).

\section{Working Memory}

Although MK-801 produces some of the memory impairments associated with hippocampal-system lesions (i.e., spatial WM and RM acquisition), this similarity is incomplete. In extensively trained rats, hippocampalsystem lesions produce severe WM deficits, whereas MK-801 does not (Jarrard, 1988; Olton \& Papas, 1979). Whereas lesion experiments show a dissociation between WM and RM performance in trained animals, the present study shows a consistent association between WM and RM in rats given MK-801. No dose of MK-801 was found to impair short-term WM selectively. Furthermore, even a 2-h delay interposed between the first two and the last two correct choices in the maze did not produce a selective WM deficit. Thus, WM performance must depend upon a different mechanism than NMDA-dependent synaptic plasticity.

Naloxone and WM. Opiate-dependent synaptic plasticity is independent of NMDA receptors and, in principle, could have provided a mechanism for WM in rats given MK-801. LTP induction in mossy fiber-CA3 synapses is blocked by naloxone (Derrick \& Martinez, 1988) but not by NMDA antagonists (Harris \& Cotman, 1986). However, naloxone facilitates performance in the radial maze (Gallagher, 1985). Thus, opiate-dependent plasticity by itself is neither necessary nor sufficient for either spatial WM or RM in normal animals. In the present study, high doses of naloxone combined with MK-801 had no effect on WM in trained rats. Thus, WM depends on neither NMDA- nor opiate-associated synaptic plasticity. Although higher doses of naloxone together with MK-801 may have impaired WM, such doses of naloxone would produce many nonspecific effects and would therefore be difficult to interpret (Sawynok et al., 1979). The present results further suggest that spatial WM performance does not require any known form of hippocampal LTP.

Is WM performance the same as learning? MK- 801 selectively impaired the acquisition, but not the retention, of WM. These results, together with the pronounced and selective deficit in spatial WM produced by fornix lesions in well-trained rats, have important implications for the organization of memory systems. Learning to perform the WM task requires functioning NMDA receptors. In contrast, subsequent WM performance, which requires daily reacquisition of memory concerning, for example, the specific arms entered during a trial, does not require functioning NMDA receptors. If WM is reacquired on each trial, and if memory acquisition requires NMDA receptors, why is WM performance not impaired by MK-801?

Perhaps MK-801 impairs the storage of spatial information and WM performance did not require spatial memory storage during the retention tests (see results of the spatial probe tests, above). In this view, the rats given MK-801 during the retention test performed the WM procedure by storing, for example, intramaze cues and not the spatial position associated with each arm of the maze. This might also explain the gradual acquisition of the WM task by the MK rats. If we assume that learning to use nonspatial cues to solve the WM task requires more trials than does learning to use spatial cues and is aided by the use of spatial cues, then perhaps by the end of acquisition training the SAL rats could use either set of cues, and therefore MK-801 did not impair WM performance. This hypothesis can be tested by forcing rats to perform a WM task using spatial cues, such as in the Morris water maze. 
Alternatively, both initial acquisition and well-learned performance of WM may require NMDA receptor activation, but in different amounts. In this view, WM retention was impaired by high doses of MK-801 because fewer NMDA receptors are required for such performance than for initial acquisition. This argument is diminished by the association of WM and RM errors, together with the nonspecific behavioral impairment produced by these high doses in the drug probes described above. Rather, the absence of a dissociation in memory performance suggests that the impairment of WM by high doses of MK-801 was a side effect of nonspecific behavioral impairments, and the paradox remains.

The following argument assumes that the hippocampal system is required for relational (e.g., spatial) memory (Eichenbaum \& Cohen, 1988; Sutherland \& Rudy, 1989), and that MK-801 impairs spatial learning by blocking synaptic plasticity in the hippocampus. Conceptually, trialdependent WM "acquisition" can be distinguished from the more gradual acquisition of RM knowledge such as that of win-shift strategies or spatial environments that are necessary for performing WM. The acquisition of relational $\mathrm{RM}$ requires the construction of representations (e.g., a cognitive map; O'Keefe \& Nadel, 1978), which differs from operations upon these structures (e.g., activation; Anderson, 1983). Adopting this view, we argue that all WM performance depends upon the existence of some kind of RM representations, but WM does not require the construction of these representations. In this view, the data suggest the following hypotheses. First, tasks that require the hippocampus for acquiring RM performance (e.g., spatial tasks) will require NMDA receptor activation for constructing RM representations. Second, the hippocampus will be needed for performing WM operations that use these "hippocampus-dependent" RM representations. Third, NMDA receptor function is not required for WM operations, except that without a representational substrate, WM cannot function. Finally, WM tasks should be unimpaired by hippocampal lesions when the representation to be operated upon does not require the hippocampus for its construction (e.g., cued WM at short delays). Thus, both hippocampal lesions and NMDA receptor blockade impair RM acquisition because these receptors in the hippocampus are required for constructing spatial representations. Once the spatial representation is constructed, the hippocampus may no longer be required for RM performance, but it continues to be necessary for WM. Thus, the hippocampus has two distinct functions: representation construction (RM) and some kind of activation or "flagging" function (WM). One possible locus for this WM function may be the mossy fiber-CA3 synapses that demonstrate an NMDAindependent form of LTP (Harris \& Cotman, 1986). Alternatively, this WM mechanism may require cholinergic input to the hippocampus from the septum (Shapiro et al., 1989). Preliminary results of an ongoing study suggest that MK-801 combined with atropine sulfate increases WM errors but not RM errors.
Note that this view may not seem to predict that WM performance would improve while RM errors remained constant, as shown here, but would predict that the acquisition of WM performance should depend on, and therefore follow, RM acquisition. However, the RM task tested explicitly here was only one component of the RM required for the task. Other RM components, such as the presence of food at the end of maze arms, were learned by the rats during the shaping procedure. The explicit (baited/unbaited) RM task was more difficult than the WM task for the SAL rats (Figures $1 \mathrm{~A}$ and $1 \mathrm{~B}$ ). Indeed, by the third block of training, the SAL rats performed almost perfectly on the WM task but made more than 2 RM errors. This dissociation shows that normal rats distinguished between entered and unentered arms even when they did not discriminate between baited and unbaited arms. Both of these discriminations require the rats to identify and remember individual arms, which remained constant from trial to trial and are thus operationally RM procedures. The knowledge about the existence (and possibly the positions) of the individual arms may provide a "primal sketch"-an impoverished representation of the environment that includes the difference among the eight maze arms-that is used in WM operations even when the RM discrimination is not yet learned. ${ }^{5}$ According to the present view, the MK rats slowly constructed this impoverished representation of the maze in $\mathrm{RM}$, and used it to perform the WM task. From this perspective, the complete representation, which included the baited/unbaited discrimination, was not constructed until several trials after MK-801 withdrawal.

This view also provides an explanation for the different levels of WM performance shown by the rats given MK-801 during acquisition. The "tolerant" rats performed better than the "nontolerant" rats from the first block of acquisition testing (Figure 1C). Therefore, the tolerant rats may have learned to discriminate maze arms during the initial shaping procedure more thoroughly than did the nontolerant subgroup. According to the current view, the tolerant rats had already begun to construct the primal sketch of the maze when MK-801 was administered. The drug impaired further construction of this sketch, but did not block it completely. The nontolerant group had acquired less of this sketch when drug trials began, but had the same learning slope as the tolerant group. After MK-801 withdrawal, the nontolerant group completed the primal sketch and performed the WM task as well as the tolerant group. The rapid improvement of WM performance after MK-801 withdrawal suggests that this final construction process can occur very quickly when the NMDA receptor system is available.

\section{Caveats}

The above discussion was based implicitly on hypotheses that attempt to link physiological data with computational models of memory implemented in neural networks (e.g., McNaughton, Leonard, \& Chen, 1989; McNaughton \& Morris, 1987). Such models store information in patterns of synaptic weights, and learning oc- 
curs by changing these weights. In particular, the properties of LTP in the hippocampus, together with results of lesion and recording studies, suggest that the hippocampus may be important for learning and memory in part because it provides the rest of the brain with a neural network containing highly plastic synapses. We have focused our interpretations on these working hypotheses. However, alternative interpretations of the data may be equally plausible, and some of these interpretations are listed below.

Hippocampal function, MK-801, and spatial memory. Because MK-801 was administered systemically, the effect of the drug cannot be localized to any one brain area, nor to any simple pharmacological effect. In the above discussion, we have focused on the effects of NMDA antagonists on synaptic plasticity and hippocampal function. However, MK-801 may affect other brain regions and other transmitter systems (e.g., dopamine metabolism; Serrano, D'Angio, \& Scatton, 1989), and these effects may have influenced the results obtained here. Furthermore, the hippocampus is not the only brain area required for spatial learning and memory. Lesions of other brain areas impair spatial learning (e.g., Kolb, Sutherland, \& Wishaw, 1983), and lesions in the hippocampal system do not always produce spatial memory deficits (Jarrard, 1988). Thus, MK-801 may have impaired spatial learning by affecting these other systems. Nonetheless, the striking similarity between the learning deficits reported here and those produced by direct infusion of the NMDA antagonist AP5 into the hippocampus, but not into visual cortical areas (Morris, Halliwell, \& Bowery, 1989), strongly suggests that the present results are indeed produced, at least in part, by antagonism of NMDA receptors in the hippocampus.

The effects of MK-801 on learning and memory reported here are almost identical to those obtained by using AP5, which blocks LTP induction at the same dose used to impair memory acquisition (Morris, 1989). MK-801 blocks LTP induction in hippocampal slices in vitro, and the present results suggest that low doses of MK-801 should also block LTP induced by learning (see Sharp et al., 1985). The above arguments assume that MK-801 blocks synaptic plasticity induction in behaving rats, but, to our knowledge, the effects of such low doses of MK-801 on in vivo LTP induction in behaving rats have not been reported. If the effects of MK-801 resemble those of AP5, then blockade of LTP in the hippocampus should parallel the impairment of memory acquisition. Future experiments will undoubtedly examine this question.

Sequential drug doses. The rats in the present study were injected repeatedly with MK-801 after the acquisition and retention tests. This chronic drug administration may have influenced the results of the subsequent behavioral tests, either by producing brain damage or by increasing or decreasing the subsequent effects of the drug. However, at the end of testing, the two groups of rats performed similarly in the long-term WM test (Ta- ble 1), although the MK group was given much more of the drug than the SAL group. Furthermore, the performance of the rats given MK-801 at this time did not differ from that during the drug probes given at the beginning of the experiment, although the baseline of normal performance did improve (compare Figure 3, SAL BL 9 and Figure 4A, Sal). Therefore, although repeated drug doses may have influenced the results obtained here, the effect was small. Nonetheless, an interaction of primary and chronic drug effects may have occurred. In any case, these interactions would not have affected the acquisition and retention results.

Strain differences. The present study used female albino Sprague-Dawley rats. In preliminary tests, a $0.2-\mathrm{mg} / \mathrm{kg}$ dose of MK-801 anesthetized a group of Sprague-Dawley females, but produced only mild ataxia in a group of Long-Evans males. This strain or sex difference may be critical for interpreting studies using MK-801.

\section{Conclusions}

The NMDA antagonist MK-801 impaired both WM and $R M$ acquisition in naive rats, but did not affect the performance of either memory task in rats trained in a radial maze task. The present data provide additional evidence that NMDA receptor activation is required for learning, but not for performing spatial memory tasks, and suggest that spatial WM storage, at least at relatively short delays, is independent of the NMDA receptor.

\section{REFERENCES}

Abraham, W. C., Mason, S. E. (1988). Effects of the NMDA receptor/channel antagonists CPP and MK801 on hippocampal field potentials and long-term potentiation in anesthetized rats. Brain Research, 462, 40-46.

Anderson, J. R. (1983). The architecture of cognition. Cambridge, MA: Harvard University Press.

BARnes, C. A. (1988). Spatial leaming and memory processes: The search for their neurobiological mechanisms in the rat. Trends in Neurosciences, 11, 163-169.

Barnes, C. A., MCNaughton, B. L. (1985). An age comparison of the rates of acquisition and forgetting of spatial information in relation to long-term enhancement of hippocampal synapses. Behavioral Neuroscience, 99, 1040-1048.

BAudry, M., LARSON, J., LYNCH, G. (1988). Long-term changes in synaptic efficacy: Potential mechanisms and implications. In $\mathbf{P}$. W. Landfield \& S. A. Deadwyler (Eds.), Long-term potentiation: From biophysics to behavior (pp. 3-72). New York: Alan Liss.

BuIss, T. V. P., LYNCH, M. A. (1988). Long-term potentiation of synaptic transmission in the hippocampus: Properties and mechanisms. In P. W. Landfield \& S. A. Deadwyler (Eds.), Long-term potentiation: From biophysics to behavior (pp. 3-72). New York: Alan Liss.

Brown, T. H., Chapman, P. F., Kairiss, E. W., Kennan, C. L (1988). Long-term synaptic potentiation. Science, 242, 724-728.

Burgard, E. C., Decker, G., \& Sarvey, J. M. (1989). NMDA receptor antagonists block norepinephrine-induced long-lasting potentiation and long-term potentiation in rat dentate gyrus. Brain Research, $\mathbf{4 8 2}$, 351-355.

Coan, E. J., Saywood, W., \& Collingridge, G. L. (1987). NK-801 blocks NMDA receptor-mediated synaptic transmission and long-term potentiation in rat hippocampal slices. Neuroscience Letters, 80 , 111-114. 
Collingridge, G. L., Kehl, S. J., \& Mclennan, H. (1983). Excitatory amino acids in synaptic transmission in the Schaffer collateralcommissural pathway of the rat hippocampus. Joumal of Physiology, $334,33-46$

Derrick, B. E., \& MARTINEZ, J. L. (1988). Naloxone blocks mossy fibre but not commissural or heterosynaptic long-term potentiation in the hippocampal CA3 region in vivo. Society for Neuroscience Abstracts, 14, 565.

DUNNETT, S. B. (1985). Comparative effects of cholinergic drugs and lesions of nucleus basalis or fimbria-fornix on delayed matching in rats. Psychopharmacology, 87, 357-363.

Eichenbaum, H., \& CoHEN, N. J. (1988). Representation in the hippocampus: What do hippocampal neurons code? Trends in Neurosciences, 11, 244-248.

Gallagher, M. (1985). Re-viewing modulation of learning and memory. In N. M. Weinberger, J. L. McGaugh, \& G. Lynch (Eds.), Memory systems of the brain (pp. 311-334). New York: Guilford.

GreEN, E. J., \& GreENough, W. T. (1986). Altered synaptic transmission in dentate gyrus of rats reared in complex environments: Evidence from hippocampal slices maintained in vitro. Journal of Neurophysiology, 55, 739-750.

HarRIs, E. W., Cotman, C. W. (1986). Long-term potentiation of guinea pig mossy fiber responses is not blocked by $\mathrm{N}$-methyl-Daspartate antagonists. Neuroscience Letters, 70, 132-137.

Hirotsu, I., Hori, N., Katsuda, N., \& Ishihara, T. (1989). Effect of anticholinergic drug on long-term potentiation in rat hippocampal slices. Brain Research, 482, 194-197.

JARRARD, L. E. (1988). Selective hippocampal lesions and behavior: Implications for current research and theorizing. In R. L. Isaacson \& K. H. Pribram (Eds.), The hippocampus (Vol. 4, pp. 93-126). New York: Plenum

Johnston, D., Hopkins, W. F., \& Gray, R. (1989). The role of norepinephrine in long-term potentiation at mossy-fiber synapses in the hippocampus. In J. H. Byme \& W. O. Berry (Eds.), Neural models of plasticity (pp. 307-328). San Diego: Academic Press.

Kemp. J. A., Priestly, T., \& WoOdruff, G. N. (1986). MK-801, a novel, orally active anticonvulsant, is a potent, non-competitive N-methyl-D-aspartate receptor antagonist. British Journal of Pharmacology Proceedings (Suppl. 89), 353.

Kolb, B., Sutherland, R. J., \& Whishaw, I. Q. (1983). A comparison of the contributions of the frontal and parietal association cortex to spatial localization in rats. Behavioral Neuroscience, 97, 13-27.

LYNCH, G. (1986). Synapses, circuits, and the beginnings of memory. Cambridge, MA: MIT Press.

MARR, D. (1982). Vision. San Francisco: W. H. Freeman.

McNaughton, B. L., Barnes, C. A., Rao, G., Baldwin, J., \& RasMUSSEN, M. (1986). Long-term enhancement of hippocampal synaptic transmission and the acquisition of spatial information. Journal of Neuroscience, 6, 563-571.

McNaughton, B. L., Leonard, B., \& Chen, L. (1989). Corticalhippocampal interactions and cognitive mapping: A hypothesis based on reintegration of the parietal and inferotemporal pathways for visual processing. Psychobiology, 17, 236-246.

MCNAUGhTON, B. L., \& MoRRIs, R. G. M. (1987). Hippocampal synaptic enhancement and information storage within a distributed memory system. Trends in Neurosciences, 10, 408-415.

Mishkin, M. (1982). A memory system in the monkey. Philosophical Transactions of the Royal Society, Series B, 298, 85-95.

MoRRIS, R. G. M. (1989). Synaptic plasticity and learning: Selective impairment of learning in rats and blockade of long-term potentiation in vivo by the n-methyl-d-aspartate receptor antagonist AP5. Journal of Neuroscience, 9, 3040-3057.

Morris, R. G. M., ANDerson, E., LynCh, G. S., \& Baudry, M. (1986). Selective impairment of learning and blockade of long-term potentiation by an $\mathrm{N}$-methyl-D-aspartate receptor antagonist, AP5. Nature, 319, 774-776.

MORRIS, R. G. M., HALLIWELl, R. F., \& BoWERY, N. (1989). Synaptic plasticity and learning: $\Pi$. Do different kinds of plasticity underlie different kinds of learning? Neuropsychologia, 27, 41-59.

Muller, D., Larson, J., \& LYNCH, G. (1989). The NMDA receptor- mediated components of responses evoked by patterned stimulation are not increased by long-term potentiation. Brain Research, 477, 396-399.

O'KeEFE, J. A., \& NADEL, L. (1978). The hippocampus as a cognitive map. Oxford: Clarendon.

O'Keefe, J. A., \& Speakman, A. (1987). Single unit activity in the hippocampus during a spatial memory task. Experimental Brain Research, 68, 1-27.

Olney, J. W., Labruyere, J., \& Price, M. T. (1989). Pathological changes induced in cerebrocortical neurons by phencyclidine and related drugs. Science, 244, 1360-1362.

OLton, D. S. (1983). Memory functions and the hippocampus. In W. Seifert (Ed.), Neurobiology of the hippocampus (pp. 335-374). New York: Academic Press.

Olton, D. S., Becker, J. T., \& Handelmann, G. E. (1979). Hippocampus, time, and memory. Behavioral \& Brain Sciences, 2 , 313-365.

Olton, D. S., \& PAPAS, B. C. (1979). Spatial memory and hippocampal function. Neuropsychologia, 17, 669-682.

Olton, D. S., \& SAmuelson, R. J. (1976). Remembrance of places passed: Spatial memory in rats. Journal of Experimental Psychology: Animal Behavior Processes, 2, 97-116.

Pontecorvo, M. J., \& Clissold, D. B. (1988). N-methyl-D-aspartate antagonism and working memory performance. Society for Neuroscience Abstracts, 14, 248.

Rawlins, J. N. P. (1985). Associations across time: The hippocampus as a temporary memory store. Behavioral \& Brain Sciences, 8 , 479-496.

Robinson, G. S., JR., Crooks, G. B., JR., Shinkman, P. G., \& GalLAGHER, M. (1989). Behavioral effects of MK-801 mimic deficits associated with hippocampal damage. Psychobiology, 17, 156-164.

Sawynok, J., Pinsky, C., \& La Bella, P. S. (1979). On the specificity of naloxone as an opiate antagonist. Life Sciences, 25, 1621-1632.

Serrano, A., D'ANGio, M., \& SCATton, B. (1989). NMDA antagonists block restraint-induced increase in extracellular DOPAC in rat nucleus accumbens. European Journal of Pharmacology, 162, 157-166.

Shapiro, M. L., Simon, D. K., Olton, D. S., Gage, F. H., NilsSON, O., \& BJORKLUND, A. (1989). Intrahippocampal grafts of fetal basal forebrain tissue alter place fields in the hippocampus of rats with fimbria-fornix lesions. Neuroscience, 32, 1-18.

Sharp, P. E., McNaughton, B. L., \& Barnes, C. A. (1985). Enhancement of hippocampal field potentials in rats exposed to a novel, complex environment. Brain Research, 339, 361.

Staubl, U., Thibault, O., Dilorenzo, M., Lynch, G. (1989). Antagonism of NMDA receptors impairs acquisition but not retention of olfactory memory. Behavioral Neuroscience, 103, 54-60.

Sutherland, R. J., ArNold, K. A., \& Rodriguez, A. R. (1987). Anterograde and retrograde effects on place memory after limbic or diencephalic damage. Society for Neuroscience Abstracts, 13, 1066.

SUTHERLAND, R. J., \& RUDY, J. W. (1989). Configural association theory: The role of the hippocampal formation in learning, memory, and amnesia. Psychobiology, 17, 129-144.

SwartzWelder, H. S. Ferrari, C., Anderson, W. W., \& Wilson, W. A. (1989). The drug MK-801 attenuates the development, but not the expression, of long-term potentiation and stimulus train-induced bursting in hippocampal slices. Neuropharmacology, 28, 441-445.

Tonkiss, J., Morris, R. G. M., \& Rawlins, J. N. P. (1988). Intraventricular infusion of the NMDA antagonist AP5 impairs performance on a non-spatial operant DRL task in the rat. Experimental Brain Research, 73, 181

Troupin, A. S., Mendius, J. R., Cheng, F., \& Risinger, M. W. (1986). MK-801. In B. S. Meldrum \& R. J. Porter (Eds.), Current problems in epilepsy: 4. New anticonvulsant drugs (pp. 191-201). London: Libby.

\section{NOTES}

1. In this context, tolerant and nontolerant are meant only to describe the behavioral effects of MK-801, and are not intended to imply any known pharmacological mechanism. 
2. Studies now under way have shown that rats given the same MK-801 treatment described here can learn to discriminate baited from unbaited arms when the arms are marked by curtains placed at the entrance to each arm.

3. The Wilcoxon signed-ranks test was used here because the rats made no WM errors in the naloxone tests, hence there was no variance for an ANOVA to test.

4. Norepinephrine (NE)-induced LTP in dentate is blocked by AP5, so it is dependent upon the NMDA channel (Burgard, Decker, \& Sarvey, 1989). However, LTP in CA3 may depend upon NE (Johnston,
Hopkins, \& Gray, 1989), and acetylcholine may affect LTP induction in CA1 (Hirotsu, Hori, Katsuda, \& Ishihara, 1989). These and other neurotransmitters may be part of a mechanism for storing WM information.

5. The primal sketch concept used here is analogous to the description of visual processing proposed by Marr (1982).

(Manuscript received August 31, 1989; revision accepted for publication February 6, 1990.)

\title{
Notices and Announcements
}

\author{
20th Annual Meeting of the Society for Computers in Psychology \\ New Orleans, Louisiana \\ November 15, 1990
}

The 20th Annual Meeting of the Society for Computers in Psychology will be held at the Hyatt Regency Hotel in New Orleans on November 15, 1990. The meeting will include presentations, discussions, and times to preview software and hardware. All areas of psychology are featured, including research, education, clinical practice, and industrial applications.

For further information, contact Sarah Ransdell, Department of Psychology, University of Maine, Orono, ME 04469 (BITNET address RANSDELL@MAINE) or C. Michael Levy, Department of Psychology, University of Florida, Gainesville, FL 32611 (BITNET address MLEVY@UFFSC).

\section{1st Annual Meeting of the Psychonomic Society \\ New Orleans, Louisiana \\ November 16-18, 1990}

The 31st Annual Meeting of the Psychonomic Society will be held in New Orleans, November 16-18, 1990. The meetings will begin Friday morning and continue until Sunday at noon. The headquarters hotel will be the Hyatt Regency.

The program and hotel reservation cards will be mailed to members and associates in September. A copy of the program will be published in the November issue of the Bulletin of the Psychonomic Society.

For further information, please contact the secretary-treasurer of the Society: Cynthia H. Null, Psychology Department, College of William \& Mary, Williamsburg, Virginia 23185 (Telephone: 804-221-3882; BITNET: WPSLCHN@WMMVS). 